Bie, J. de, Kijlstra, N.B., Daemen, B.J.G., Bouvy, M.L. The development of quality indicators for community pharmacy care. BMJ Quality and Safety: 2011, 20(8), 666-671

\begin{tabular}{|l|l|}
\hline $\begin{array}{l}\text { Postprint } \\
\text { Version }\end{array}$ & 1.0 \\
\hline Journal website & $\frac{\text { http://qualitysafety.bmi.com/content/20/8/666.abstract?sid=b20aeff8-66d2-48b5- }}{\underline{\text { aa02-edef1ad4bcc1 }}}$ \\
\hline Pubmed link & $\underline{\text { http://www.ncbi.nlm.nih.gov/pubmed/21389023 }}$ \\
\hline DOI & $10.1136 /$ bmjqs.2010.045237 \\
\hline
\end{tabular}

This is a NIVEL certified Post Print, more info at http://www.nivel.eu

\title{
The development of quality indicators for community pharmacy care
}

\author{
J DE BiE ${ }^{1}$, N B KiJlstra ${ }^{2}$, B J G DAEMEN ${ }^{3}$, M L BOUVY ${ }^{1,4}$ \\ ${ }^{1}$ SIR Institute for Pharmacy Practice and Policy, Leiden, The Netherlands \\ ${ }^{2}$ Dutch Health Care Inspectorate, The Netherlands \\ ${ }^{3}$ KNMP, Royal Dutch Pharmacists Association, The Netherlands \\ ${ }^{4}$ Division of Pharmacoepidemiology and Clinical Pharmacology, Utrecht Institute for \\ Pharmaceutical Sciences (UIPS), Utrecht University, Utrecht, The Netherlands
}

\begin{abstract}
Aims To develop a national system of quality indicators for community pharmacy care, reported by community pharmacies.

Methods After preliminary validation, an online consensus study was conducted. Pharmacy practice experts (round 1) and practising pharmacists (round 2) were approached. Face-validity scores for risk of harm and quality of care were obtained, as well as data on feasibility and clarity. To field test the data collection a random sample of 30 community pharmacies reported on the indicators, and were visited by a healthcare inspector to discuss practical and interpretation issues.
\end{abstract}

Results After preliminary validation of 159 topics, 53 indicators were rated in round 1 by 14 of the 16 experts who were approached. The resulting 48 indicators were rated by 76 pharmacists of the 150 pharmacists who were approached (response 50\%). Of the 48 indicators in the second round, 33 (69\%) were rated face valid (median relevance score $\geq 7$ ) for risk of harm to patients, while 43 (90\%) were rated face valid for quality of care. In the field test the participating pharmacies reported on the resulting set of 44 indicators. This resulted in an adjusted set of 42 indicators. The set contains indicators on patient counselling (6), clinical risk management (10), compounding (7), dispensing (3), monitoring of medication use (11) and quality management (5).

Conclusions A set of 42 quality indicators was developed for community pharmacy care. It is expected that this will have a positive impact on quality and safety of community pharmacy care in the Netherlands. 


\section{INTRODUCTION}

The quality and safety of healthcare systems has received increasing attention from policy makers and researchers in the past decade. There have been several studies on patient safety that have raised awareness because of the frequency and nature of medication-related errors. ${ }^{1-5}$ The studies suggest that, internationally, approximately $5 \%$ of hospital admissions are directly related to the side effects of medication. ${ }^{3-5}$ Moreover it is suggested that almost half of these admissions could be prevented. ${ }^{4}$ Because of the major role played by pharmacies in the provision of care surrounding medication, a focus on medication safety and quality of care in pharmacies may increase patient safety.

Assessment of quality plays an important role in its improvement. ${ }^{6}$ Quality indicators can be used in this assessment. Indicators are measurable items referring to structures, processes or outcomes of care. ${ }^{67}$ In 2003 the Dutch Healthcare Inspectorate first introduced quality indicators for public reporting on hospitals. ${ }^{8}$ These indicators were developed by the Inspectorate to help detect unsafe situations. Moreover the indicators aimed to increase transparency for healthcare providers, patients and health insurance companies. Positive results in quality improvement were reported, for instance in registration of pressure ulcers. ${ }^{9}$ More recently the Ministry of Health decided to introduce quality indicators for all sectors of healthcare, including community pharmacy. In addition to the quality indicators developed by the Inspectorate, these will include measures of quality from a consumer perspective.

Although indicators focusing on preventable drug-related morbidity in primary care have been developed in the USA, Canada and the UK ${ }^{10-13}$ and for community pharmacies in Portugal, ${ }^{14}$ to the authors' knowledge there is no integral set of quality indicators for different aspects of community pharmacy care. Therefore the Dutch Healthcare Inspectorate and the Royal Dutch Pharmacists Association (KNMP) aimed to develop quality indicators to provide an indication of the risk of harm to patients and quality of care in community pharmacies. This paper describes the development of these quality indicators and the views of pharmacists about their development.

\section{METHODS}

\section{Setting}

In 2008 the Netherlands had 1948 community pharmacies, and on average dispensed 85000 prescriptions per year and had an average population of 7800 patients. Every pharmacy had an average of 5.9 pharmacy technicians, 1 managing pharmacist, 0.5 second pharmacists and 1.7 other pharmacy employees.15

\section{Aim of indicators and criteria used in their development}

The indicators were designed to provide self-reported data on the structure, process and outcome of care at the level of a community pharmacy. Structure indicators mainly consist of questions on common practice, working agreements and protocols in the pharmacy. Process indicators include questions on the actual adherence to working agreements and protocols, and mainly relate to searches performed using the pharmacy information system and data available from separate records kept by pharmacies (such as error registration data and analysis of pharmacy compounding). Because of the limited data available on clinical outcomes in community pharmacies, 
outcome indicators were based on dispensing data, for example numbers of patients who had received a (un)desired combination of medications. If possible, structure, process and outcome indicators were included.

Throughout the development of the indicators the major criteria for inclusion were relevance for pharmacy practice and validity for quality of care or risk of harm to patients. Relevance for quality of care and risk of harm to patients was rated in rounds 1 and 2. Rating ranged from 1 (little relevance) to 9 (very relevant). Indicators with an overall median rating of 7, 8 or 9 in round 2, without

disagreement, were considered face valid. Disagreement was defined as $25 \%$ or more scores at both ends of the rating scale (1-3). Clarity of wording, availability of data and qualitative comments were also considered. Decisions on inclusion, adjustment or exclusion were made by the working group after discussion following each step.

\section{Design of the study}

After preliminary validation of an initial set of indicators, a two-round consensus study was carried out, followed by a field test of the data collection. Similar methodology was used previously in the development of preventable drug-related morbidity and prescribing indicators.10-13

\section{Preliminary validation}

An initial set of indicators was generated after a search of the international literature, pharmacy practice and prescribing guidelines, and pharmacy-related or drug-related indicators from other initiatives.10-13 16-19 Inclusion was based on expert opinion and, if applicable, recent prescription rates or other data to assess prevalence in daily clinical practice. The working group performed a preliminary validation focusing on the relevance to quality of care and risk of harm to patients in community pharmacy practice.

\section{Round 1: pharmacy practice experts}

An email questionnaire was sent to a panel of pharmacy practice experts $(n=16)$, consisting of 14 pharmacists and two pharmacy technicians. All experts were active in pharmacy practice care and were also involved in various fields, such as professional education, research and guideline development. Each expert rated at least eight randomly allocated mandatory indicators. They were invited to provide comments and suggestions for these indicators or any others in the set.

\section{Round 2: pharmacists}

One hundred and fifty-one community pharmacists were invited to participate in round 2 using a web-based questionnaire. All pharmacists were involved in daily practice and were drawn from a sample that had been involved in earlier educational or research projects of the working group. In addition to rating the indicators and commenting on the clarity of the wording and feasibility of data collection, background information and pharmacists' views on quality indicators were sought. Qualitative comments could be added at the end of the questionnaire. If an indicator failed to meet the set criteria for face validity, it could only be included after extensive rewording.

\section{Field test}

To test the feasibility of data collection in daily pharmacy practice, 30 community pharmacies were randomly approached to take part in a field test. These pharmacies 
Bie, J. de, Kijlstra, N.B., Daemen, B.J.G., Bouvy, M.L. The development of quality indicators foj community pharmacy care. BMJ Quality and Safety: 2011, 20(8), 666-671

were asked to provide data on the indicators. All participating pharmacies were visited by a senior healthcare inspector. During this visit practical aspects of data collection and interpretation of the indicators were discussed.

\section{RESULTS}

The development of the indicators is summarised in figure 1.

\section{Preliminary validation}

Of an initial set of 159 topics and indicators, 53 indicators were assessed as sufficiently relevant for risk of harm and quality of care in a community pharmacy setting. These were used as input for round 1 . The set included indicators on patient counselling, clinical risk management, compounding, dispensing of medication, monitoring of medication use and quality management.

\section{Round 1: email consultation of experts}

Of the 16 experts approached, 12 pharmacists and two pharmacy technicians responded. Ratings, comments and suggestions by the experts were discussed by the working group, and if needed, adjustments were made. This resulted in the inclusion of 35 indicators without modification, five indicators with modification, two indicators that were split in two (resulting in four indicators), three indicators combined into one indicator, eight excluded indicators and three additional indicators. Reasons for exclusion of indicators included low prevalence, low risk and overlap between indicators. This resulted in a modified set of 48 indicators for input in round 2.

\section{Round 2: web-based consultation of pharmacists}

Of the 151 pharmacists invited to participate in the second round, 91 (60\%) completed the web-based questionnaire partially (23) or completely (68). After exclusion of respondents who rated less than eight indicators, the data of 76 pharmacists were analysed (response 50\%). The pharmacists had an average age of 44 years with 16 years' experience in community pharmacy practice; $63 \%$ was women.

Table 1 summarises the pharmacists' views about the quality indicators. Almost all pharmacists were positive about the development and use of quality indicators. A large majority felt providing data on quality indicators was feasible for their own pharmacy. A third of respondents expected negative outcomes from providing data on quality indicators to the Healthcare Inspectorate (table 1).

\section{[TABLE 1]}

Agreement between pharmacists was reached for all 48 indicators for risk of harm to patients. For quality of care, agreement was reached for 45 of the 48 indicators (94\%). Of the 48 indicators, 33 (69\%) were rated face valid (median relevance score $\geq 7$ ) for risk of harm to patients, while $43(90 \%)$ were rated face valid for quality of care (figure 2).

The percentage of pharmacists who felt the wording of the indicators was not sufficiently clear ranged from 0 to $15.3 \%$. The wording of four indicators was judged as not sufficiently clear by more than $10 \%$ of the pharmacists. The percentage of pharmacists who did not expect to be able to generate data for a given indicator 
ranged from 0 to 39\%. For five indicators, more than $10 \%$ of pharmacists did not expect to be able to generate the required data.

After round 2, 28 out of 48 indicators were included without adjustments (or with only minor adjustments), 14 indicators were included with adjustments, one indicator was split in two and five indicators were excluded. Reasons for exclusion of indicators included lack of face validity for quality of care or risk of harm and expected problems with either uniform data collection or interpretation. This resulted in an adjusted set of 44 indicators for the field test.

\section{Field test}

Of the 30 pharmacies that were invited to participate in the field test, 21 finished the data collection timely (70\%) and were visited by an inspector. Reasons for nonresponse were time limitations, sick leave taken by the pharmacists or other staff, and other unforeseen circumstances. Pharmacists estimated spending a median of $3 \mathrm{~h}$ on the collection. Following the field test, four indicators were excluded because of expected problems with (uniform) data collection and/or interpretation. Twentyseven indicators were included unchanged, and adjustments were made to the remaining indicators. This resulted in a final set of 42 quality indicators, as presented in table 2. The set contains indicators on patient counselling (6), clinical risk management (10), compounding (7), dispensing of medication (3), monitoring of medication use (11) and quality management (5). Of the indicators, 13 are structure indicators, 18 process indicators and 11 outcome indicators of care.

\section{[TABLE 2]}

\section{DISCUSSION}

A national system of quality indicators for community pharmacy care, as reported by community pharmacies, was developed using a consensus study. The set covers a wide range of aspects of community pharmacy care. Ninety per cent of all indicators met the criteria for (face) validity for quality of care. Just over two-thirds were considered (face) valid for risk of harm to patients. Overall, therefore, the indicators may have a higher validity for quality of care than for potential risk of harm. The indicators are based on self-reported data by community pharmacists. Indicators from a patient's perspective were not included because of the parallel development of a consumer quality index for community pharmacies in the Netherlands.20 In the future it is expected that both types of indicators will be presented together to improve transparency of care for patients.

In the final set of indicators the proportion of structure indicators (13 of 42) —often relating to questions on existing working arrangements-was larger than anticipated at the start. In many cases formulating a measurable indicator at a process or outcome level was not feasible. This was mainly because of differences in organisation of care and availability of data in pharmacy information systems. As the presence of these indicators is an incentive to improve documentation of care, it is expected that over the years, more structure indicators may be substituted by process and outcome indicators.

The field test indicated that pharmacies should be able to provide most of the data needed for the current set of indicators. However, national data collection is expected to uncover additional practical and interpretation issues. Moreover, it is essential that 
this study is followed up by further testing of the predictive value, sensitivity and specificity of the indicators for quality of care or risk of harm to patients. In addition, the effectiveness of using the indicators under the supervision of the Healthcare Inspectorate and whether they result in quality improvement in practice requires further research.

The introduction of these indicators is an important step towards increased transparency of care for patients, other healthcare providers and health insurance companies. Although the indicators in this set are not measured through patient experience, their outcomes are relevant for patients. It is increasingly recognised that open communication of outcomes of care increases the focus of healthcare providers on quality and safety. A key issue is the acceptance of the indicators by healthcare providers. This project attempted to increase acceptance by involving different groups of experts and practising pharmacists from the start. In general, the views of the responding pharmacists on the introduction and use of the indicators seemed positive.

It is possible that pharmacists with more reservations on this subject chose not to participate in the study. Although the reporting of the indicators was compulsory, it is promising that $94 \%$ of the community pharmacies in the Netherlands provided information on the indicators in the first round of the national data collection within the given time period. Future acceptance will depend on many factors, such as the feedback given on the indicators, the interpretation of the data by the Healthcare Inspectorate and the appropriate use of the indicators. For instance, a discussion on the appropriateness of using such indicators for decisions on remuneration of services by health insurance companies is ongoing in the Netherlands.

These indicators can be adopted as a starting point for the development of quality and safety indicators for pharmacies in other countries. A core set of indicators used to assess pharmacy care in different countries, such as within the European Union, may be helpful for comparison purposes. Although the current set may be a good starting point, further international consensus would be needed.

\section{CONCLUSION}

A set of 42 quality indicators was developed for community pharmacy care. It is expected that this will have a positive impact on quality and safety of community pharmacy care in the Netherlands.

\section{ACKNOWLEDGMENTS}

For their extensive work on the quality indicators in the working group we would like to acknowledge R.J. Bruinink and F. Steenhuisen, senior health care inspectors. We would like to thank M. Teichert of the Royal Dutch Pharmacists Association for her work on the searches underlying part of the quality indicators.

\section{FOOTNOTES}

Competing interests None declared.

Provenance and peer review Not commissioned; externally peer reviewed. 
Bie, J. de, Kijlstra, N.B., Daemen, B.J.G., Bouvy, M.L. The development of quality indicators foj community pharmacy care. BMJ Quality and Safety: 2011, 20(8), 666-671

\section{REFERENCES}

1. Institute of Medicine. To err is human: building a safer health system. In: Linda T, Kohn JMC, Molla S, et al, eds. Washington D.C: National Academy Press, 1999.

2. Leape L, Berwick D, Clancy C, et al. Transforming healthcare: a safety imperative. Qual Saf Health Care 2009;18:424e8.

3. van der Hooft CS, Sturkenboom MC, van Grootheest K, et al. Adverse drug reactionrelated hospitalisations: a nationwide study in The Netherlands. Drug Saf 2006;29:161e8.

4. Leendertse AJ, Egberts AC, Stoker LJ, et al. Frequency of and risk factors for preventable medication-related hospital admissions in the Netherlands. Arch Intern Med 2008;168:1890e6.

5. Howard RL, Avery AJ, Slavenburg S, et al. Which drugs cause preventable hospital admissions to hospital? A systematic review. Br J Clin Pharmacol 2007;63:136e47.

6. Campbell SM, Braspenning J, Hutchinson A, et al. Research methods used in developing and applying quality indicators in primary care. Qual Saf Health Care 2002;11:358e64.

7. Campbell SM, Braspenning J, Hutchinson A, et al. Research methods used in developing and applying quality indicators in primary care. BMJ 2003;326:816e19.

8. Dutch Health Care Transparity Programme. Framework for quality indicators. Den Haag: Ministry of Health, 2009.

9. Health Care Inspectorate. Het resultaat telt 2008. [The result counts 2008]. Den Haag: Dutch Health Care Inspectorate, 2009.

10. Campbell SM, Cantrill JA, Roberts D. Prescribing indicators for UK general practice: Delphi consultation study. BMJ 2000;321:425e8.

11. Morris C, Cantrill J. Preventable drug-related morbidity indicators in the U.S. and U.K. J Manag Care Pharm 2002;8:372e7.

12. Morris $\mathrm{CJ}$, Cantrill JA, Hepler $\mathrm{CD}$, et al. Preventing drug-related morbidityddetermining valid indicators. Int J Qual Health Care 2002;14:183e98.

13. Morris CJ, Cantrill JA. Preventing drug-related morbiditydthe development of quality indicators. J Clin Pharm Ther 2003;28:295e305.

14. Guerreiro M. Risk management of preventable drug-related morbidity in Portuguese community pharmacies. PhD thesis. The University of Manchester, 2007.

15. Stichting Farmaceutische Kengetallen. Data en Feiten 2009, [Pharmaceutical facts and figures 2009]. Den Haag: Stichting farmaceutische Kengetallen, 2009.

16. Koninklijke Nederlandse Maatschappij ter bevordering van de Farmacie. Nederlandse Apotheeknorm 2006 [Dutch Pharmacy Standards 2006]. Den Haag: Koninklijke Nederlandse Maatschappij ter bevordering van de Farmacie, 2006.

17. Muijrers PE, Janknegt R, Sijbrandij J, et al. Prescribing indicators. Development and validation of guideline-based prescribing indicators as an instrument to measure the variation in the prescribing behaviour of general practitioners. Eur J Clin Pharmacol 2004;60:739e46.

18. Van den Berg MJ, van Roosmalen M, Braspenning JCC. De staat van de Huisartsgeneeskunde [The state of general practice]. Utrecht: Nivel, 2005.

19. Becker ML, Kallewaard M, Caspers PWJ, et al. Potential determinants of drugedrug interaction associated dispensing in community pharmacies. Drug Saf 2005;28:371e8.

20. Vervloet M, Linschoten P, van Dijk L. Consumer experiences on medication information in pharmacies and dispensing general practices. Eur J Public Health 2007;17(Suppl 2):94. 
Bie, J. de, Kijlstra, N.B., Daemen, B.J.G., Bouvy, M.L. The development of quality indicators foj community pharmacy care. BMJ Quality and Safety: 2011, 20(8), 666-671

\section{TABLES}

Figure 1 Steps taken in the development of the indicators and resulting changes.

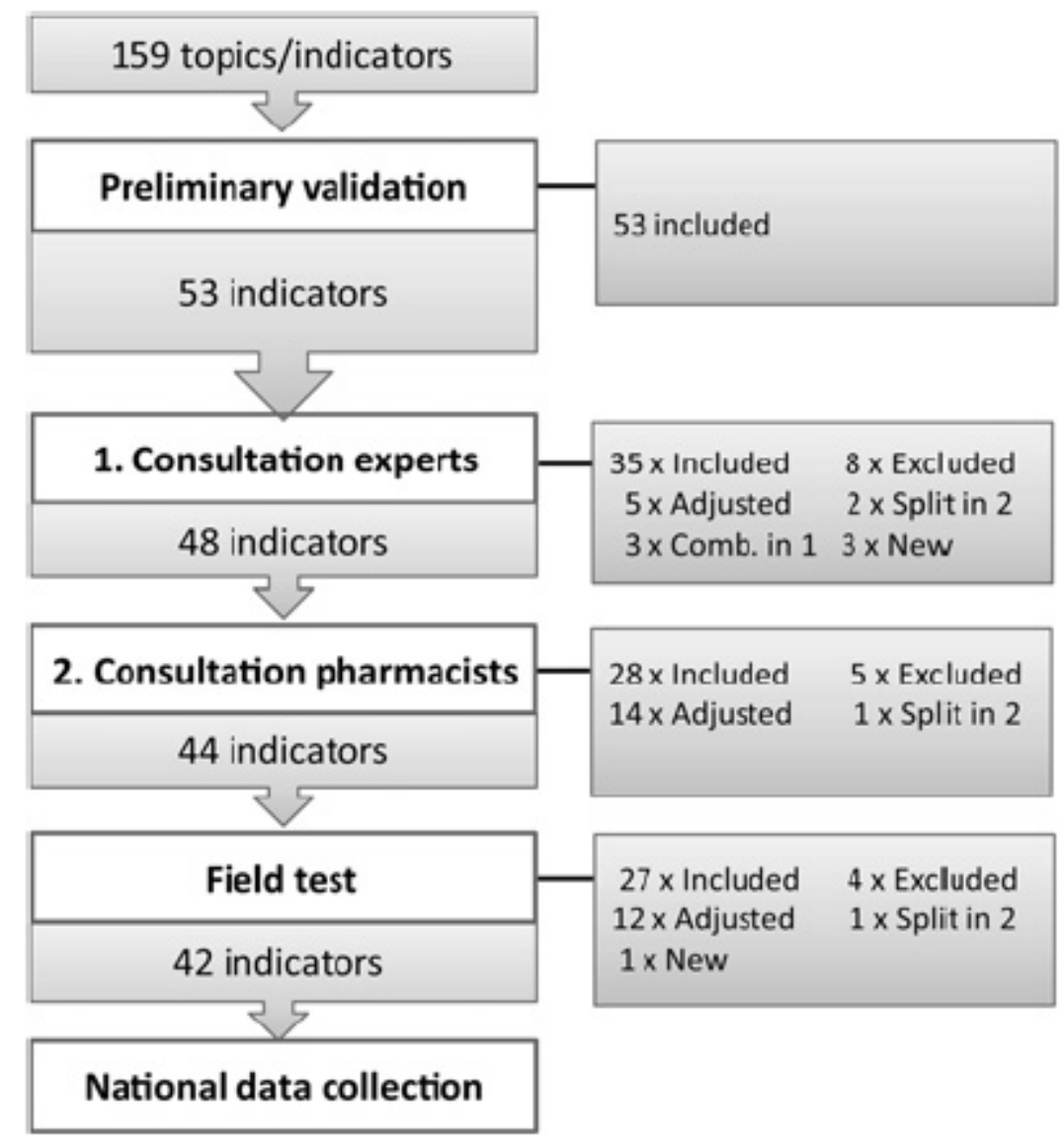

Table 1 Views of pharmacists ( $n=68)$ about quality indicators, percentages yes ((totally) in agreement with statement)

It is a positive that quality indicators are being developed for community pharmacies

The quality of care provided by community pharmacies can be measured with quality indicators $f$

The degree of risk of harm to patients can be measured with quality indicators

Providing data on quality indicators is feasible for most community pharmacies at the moment

Providing data on quality indicators is feasible for my pharmacy at the moment $\dagger$

*One missing $(n=67)$.

†Asked in negative wording; cannot be measured and is not feasible. 
Bie, J. de, Kijlstra, N.B., Daemen, B.J.G., Bouvy, M.L. The development of quality indicators fo community pharmacy care. BMJ Quality and Safety: 2011, 20(8), 666-671

Figure 2 Second round validity score; number of indicators that received the validity scores $1 \mathrm{e} 9$.

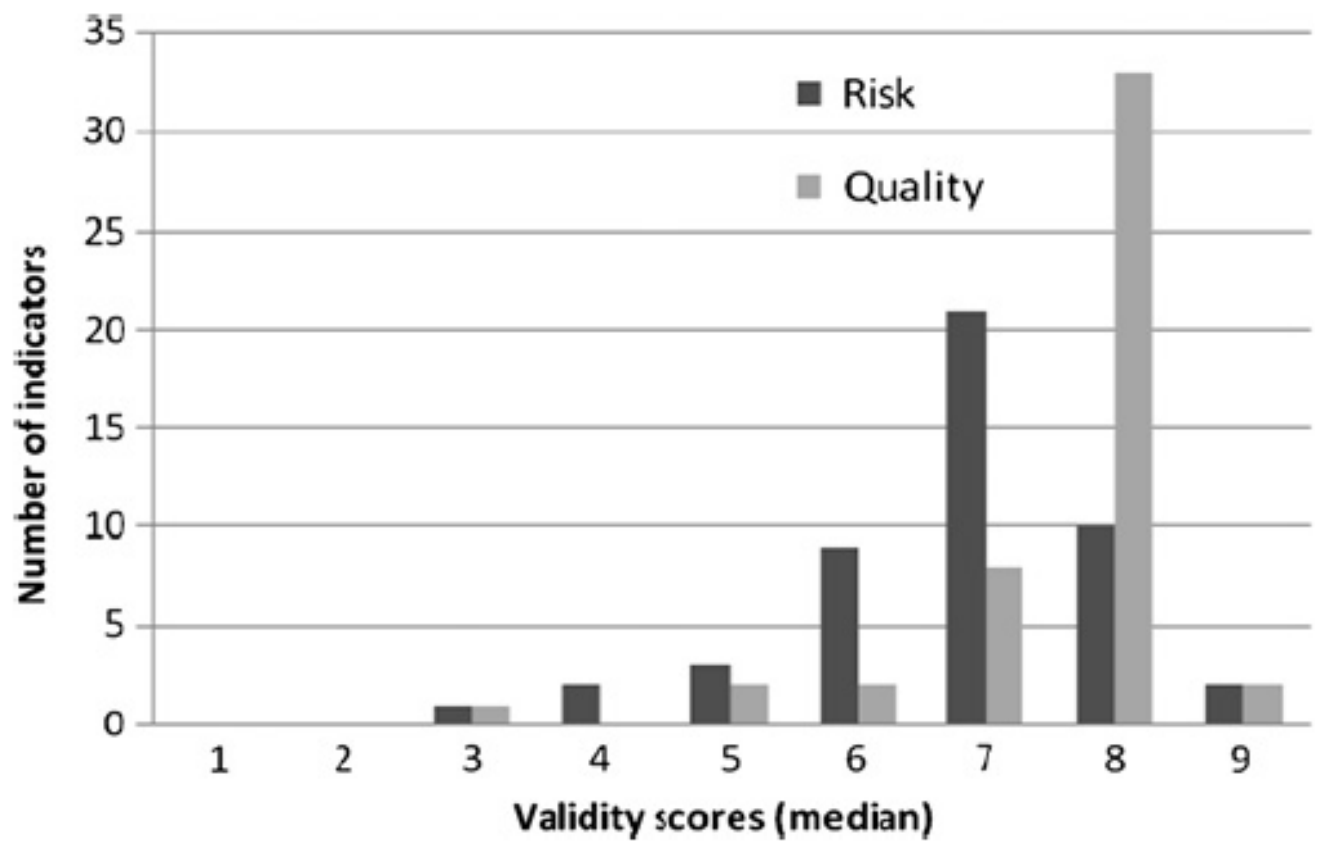


Bie, J. de, Kijlstra, N.B., Daemen, B.J.G., Bouvy, M.L. The development of quality indicators fop community pharmacy care. BMJ Quality and Safety: 2011, 20(8), 666-671

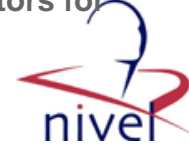

Table 2 Quality indicators for pharmaceutical care after field testing* according to subject and type of indicator

s

Patient counselling

$1 \quad$ Offering inhalation instruction to patients when inhalation medication first dispensed

2 Percentage of users of inhalation medication who received instructions for the use of inhaler devices

3 Performing checks on the correct use of blood glucose meters by diabetes patients

4 Percentage of benzodiazepine users who received verbal information about driving safety when berzodiazepines first dispensed

5 Percentage of benzodiazepine users who received verbal information about dependency with a follow-up prescription for berzodiazepines

6 Percentage of antidepressant users who received verbal information about a delay in effect when antidepressants first dispensed

Clinical risk management

7 Documentation of current medication use, prior to dispensing of medication

8 Availability of protocols concerned with asking patients about potential contraindications

9 Asking the prescriber for confirmation of suspected contraindications

10 Percentage of oral diabetes medication and/or insulin users with a documented contraindication of diabetes

11 Percentage of patients over 55 years with a documented contraindication of heart failure

12 Availability of protocols concerned with asking patients about possible drug intolerabilities Percentage of patients with a documented penicillin allergy

Documentation of the management of drug-drug interactions

Management of interactions between oral anticoagulants and co-trimoxazole

Number of patients who concurrently use anticoagulants and co-trimoxazole or miconazole

Compounding

17 Assessment of the pharmacotherapeutic rationality of extemporaneous phamacy compounding

Percentage of compounded capsules with statistical weight analysis

Percentage of compounded medicines sent to an analytical laboratory

Percentage of compounded medicines for systemic use with dose control

Percentage of compounded medicines for systemic use with drug-drug interaction controls on

individual components

22 Releasing technician compounded medicines by a phamacist before dispensing to patients

Arrangements made for checks by compounding pharmacy, in case of delegated compounding

Dispensing of medication

24 Percentage of dispensed medication with quality assurance steps applied in the dispensing process

25 Number of days that the list of medication alerts was not checked the same day by a phamacist

26 Percentage of community dwelling patients $>65$ years with medication dispensed in individualised dosage systems

Monitoring of medication use

27 Percentage of patients chronically using loop diuretics and RAS inhibitors who are dispensed NSAIDs

28 Percentage of users of COX-2 inhibitors with concomitant use of drugs that indicate ischaemic cardiovascular disease

Percentage of benzodiazepine users ( $>65$ years) who chronically use berzodiazepines

Percentage of classical NSAID users (>70 years) with gastroprotection

Percentage of patients who concurrently use nitrates and antithrombotics

Percentage of users of strong opiates who take laxatives (lactulose, magnesium oxide, bisacodyle, macrogol or sennosides)

33 Percentage of patients with inadequate dosage of inhalation corticosteroids with concurrent excessive use of bronchodilators

34 Percentage of patients with mettomine dispensed as a first prescription for oral blood glucose medication Percentage of hypnotics users who chronically use long-acting hypnotics

Percentage of dispensed third-generation chinolones

Number of medication reviews performed for patients with polypharmacy ( $\approx 5$ different types of medication)

Quality management

38 Availability of a procedure for registration of errors made during dispensing of medication, noticed within the pharmacy

39 Number of registered errors made during dispensing of medication, noticed within the phamacy

40 Year of most recent evaluation of patients' experiences

41 Number of registered complaints made by patients

42 Number of adverse drug reactions reported b the Netherlands Pharmacovigilance Centre**

$\mathrm{x}$

$\mathrm{x}$

"This table contains the subject list of the indicators; in the full set the indica tors are defined in more detail, including selection crite ria. The more detailed Dutch version is recommended, which can be obtained from the authors.

*The Netherlands Pharmacovigilance Centre operates the national registration and evaluation system for adverse drug reactions. COX-2, cyclooxygenase-2; NSAID, non-steroidal anti-inflammatory drug; RAS, renin-angiotensin system; s, structure; p, process; o outcome. 